\title{
Some totally geodesic submanifolds of the nonlinear Grassmannian of a compact symmetric space
}

\author{
Marcos Salvai*
}

\begin{abstract}
Let $M$ and $N$ be two connected smooth manifolds, where $M$ is compact and oriented and $N$ is Riemannian. Let $\mathcal{E}$ be the Fréchet manifold of all embeddings of $M$ in $N$, endowed with the canonical weak Riemannian metric.

Let $\sim$ be the equivalence relation on $\mathcal{E}$ defined by $f \sim g$ if and only if $f=g \circ \phi$ for some orientation preserving diffeomorphism $\phi$ of $M$. The Fréchet manifold $\mathcal{S}=\mathcal{E} / \sim$ of equivalence classes, which may be thought of as the set of submanifolds of $N$ diffeomorphic to $M$ and is called the nonlinear Grassmannian (or Chow manifold) of $N$ of type $M$, inherits from $\mathcal{E}$ a weak Riemannian structure. Its geodesics, although they are not good from the metric point of view, are distinguished curves and have proved to be useful in various situations.

We consider the following particular case: $N$ is a compact irreducible symmetric space and $M$ is a reflective submanifold of $N$ (that is, a connected component of the set of fixed points of an involutive isometry of $N$ ). Let $\mathcal{C}$ be the set of submanifolds of $N$ which are congruent to $M$. We prove that the natural inclusion of $\mathcal{C}$ in $\mathcal{S}$ is totally geodesic.
\end{abstract}

Mathematics Subject Classification 2000: 22C05, 53C22, 53C35, 58B20, 58D10, 58E10

Keywords and phrases: manifold of embeddings, geodesic, symmetric space, reflective submanifold

*Partially supported by FONCyT, CIEM (CONICET) and SECyT (UNC). 


\section{Introduction and statement of the result}

\subsection{Manifolds of embeddings}

Let $M, N$ be connected differentiable manifolds. If $M$ is compact and oriented and $N$ is Riemannian, then the set $\mathcal{E}$ of all embeddings of $M$ into $N$ is a Fréchet manifold [3] which has a canonical weak Riemannian metric defined as follows: If $f \in \mathcal{E}$ and $u, v \in T_{f} \mathcal{E}$ (that is, $u, v$ are smooth vector fields along $f$ ), then

$$
\langle u, v\rangle=\int_{M}\langle u(x), v(x)\rangle \omega_{f}(x),
$$

where $\omega_{f}$ is the volume element of the Riemannian metric on $M$ induced by $f$.

Let $\sim$ be the equivalence relation on $\mathcal{E}$ defined by $f \sim g$ if and only if $f=g \circ \phi$ for some orientation preserving diffeomorphism $\phi$ of $M$. The set $\mathcal{S}=\mathcal{E} / \sim$ of equivalence classes is called the nonlinear Grassmannian (or Chow manifold) of $N$ of type $M$. It is a Fréchet manifold with a weak Riemannian metric in such a way that the associated projection $\Pi: \mathcal{E} \rightarrow \mathcal{S}$ is a principal bundle with structure group $\operatorname{Diff}_{+}(M)$, and a Riemannian submersion. Cf. [5], where much more general metrics on $\mathcal{S}$ are considered.

For any $f \in \mathcal{E}$ we have the decomposition $T_{f} \mathcal{E}=\mathcal{H}_{f} \oplus \mathcal{V}_{f}$ in horizontal and vertical subspaces at $f$, where $\mathcal{V}_{f}=\operatorname{Ker}\left(d \Pi_{f}\right)$ and $\mathcal{H}_{f}$ is the orthogonal complement of $\mathcal{V}_{f}$. They consist of all the smooth vector fields along $f$ which are tangent to $f(M)$, respectively, normal, at each point of $M$.

\subsection{Reflective submanifolds}

A reflective submanifold $M$ of a Riemannian manifold $N$ is a connected component of the set of fixed points of an involutive isometry of $N$. In particular, $M$ is closed and totally geodesic in $N$. Reflective submanifolds of symmetric spaces have been extensively studied by Leung in a series of papers beginning with [4]. For instance, every complete totally geodesic connected submanifold of a simply connected space form is reflective. Also, the reflective submanifolds of $\mathbb{C} P^{n}$ are exactly, up to isometry, $\mathbb{C} P^{k}(1 \leq k<n)$ and $\mathbb{R} P^{n}$ (canonical embedding). In particular, $\mathbb{R} P^{1}$, that is, a geodesic, is not a reflective submanifold of $\mathbb{C} P^{n}$ if $n \geq 2$. 


\subsection{The nonlinear Grassmannian of a compact sym- metric space}

Let $N$ be a compact connected symmetric space and let $G$ be the identity component of the isometry group of $N$. Let $o \in N$ and let $K$ be the isotropy subgroup at $o$. We have the canonical projection $\pi: G \rightarrow N, \pi(g)=g(o)$. For the sake of simplicity, we assume further that $G$ is semisimple and $\pi$ is a Riemannian submersion, where $G$ is endowed with the Riemannian metric defined at the identity by the opposite of the Killing form.

Let $M$ be a reflective submanifold of $N$ and let $\mathcal{E}, \mathcal{S}$ be the spaces associated to $M, N$ as in 1.1. We may suppose that $o \in M$.

Let $H=\{g \in G \mid g(M)=M\}$. Since $M$ is closed in $G$, then $H$ is a closed subgroup, and hence a Lie subgroup, of $G$.

Let $\mathcal{C}$ be the set of submanifolds of $N$ which are $G$-congruent to $M$, that is, $\mathcal{C}=\{g(M) \mid g \in G\}$. We may identify $\mathcal{C} \cong G / H$.

Now we can state the main result of the paper: $\mathcal{C}$ is totally geodesic in $\mathcal{S}$. More precisely,

Theorem 1. Let $\iota: M \rightarrow N$ be the inclusion and let

$$
F: \mathcal{C} \cong G / H \rightarrow \mathcal{S}, \quad F(g H)=\Pi \circ g \circ \iota
$$

(a well-defined map). Then $(\mathcal{C}, F)$ is a totally geodesic submanifold of $\mathcal{S}$.

Remark 2. a) Although geodesics in $\mathcal{S}$ are not good from the metric point of view [6,1], they are distinguished curves. For instance, the case $M=S^{1}$, $N=S^{3}[8]$ has been useful in a characterization of the Hopf fibrations of $S^{3}$.

b) We do not know whether the Riemannian metric induced on $\mathcal{C}$ from $\mathcal{S}$ is normal with respect to $G$ (i.e., whether the canonical projection $\tilde{\pi}: G \rightarrow \mathcal{C}$ is a Riemannian submersion for some bi-invariant Riemannian metric on $G)$, but at least in the simplest case it is:

Proposition 3. Let $M$ be a refletive submanifold of $S^{n}$, that is, $M$ is a great sphere. Then the metric on $\mathcal{C}$ induced from $\mathcal{S}$ is normal.

Proof. Let $\left\{e_{i} \mid i=0, \ldots, n\right\}$ be the canonical basis of $\mathbb{R}^{n+1}$ and suppose $M=S^{n} \cap \operatorname{span}\left\{e_{i} \mid i=0, \ldots, m\right\} \cong S^{m}$. Given $0 \leq i<j \leq n$ and $t \in \mathbb{R}$, let $R_{t}^{i, j} \in S O(n+1)=G$ fixing $e_{k}$ for $k \neq i, j$ and satisfying $R_{t}^{i, j} e_{i}=$ 
$(\cos t) e_{i}+(\sin t) e_{j}$. Let $E^{i, j}=\left.\frac{d}{d t}\right|_{0} R_{t}^{i, j}$. If we take $o=e_{0}$ and call $\mathfrak{h}$ the Lie algebra of $H$, then $\mathfrak{h}^{\perp}=\operatorname{span}\left\{E^{i, j} \mid 0 \leq i \leq m<j \leq n\right\}$.

The corresponding vector fields along the inclusion $\iota: S^{m} \rightarrow S^{n}$ are

$$
V^{i, j}(q)=\left.\frac{d}{d t}\right|_{0}(\cos t) x_{i}(q) e_{i}+(\sin t) x_{i}(q) e_{j}=x_{i}(q) e_{j},
$$

where $q \in S^{m}$. Now we apply the definition (1). We compute

$$
\left|V^{i, j}(q)\right|^{2}=x_{i}^{2}(q), \quad\left\langle V^{i, j}(q), V^{k, \ell}(q)\right\rangle=\delta_{j \ell} x_{i}(q) x_{k}(q) .
$$

Since $y_{i}=\left.{ }_{\text {def }} x_{i}\right|_{S^{m}}(i=0, \ldots, m)$ are elements of the canonical orthogonal basis of spherical harmonics on $S^{m}$, we have $\left\langle V^{i, j}, V^{k, j}\right\rangle=0$ if $i \neq k$. Besides,

$$
(m+1) \int_{S^{m}} y_{i}^{2}(q) \omega_{\iota}(q)=\sum_{s=0}^{m} \int_{S^{m}} y_{s}^{2}(q) \omega_{\iota}(q)=\int_{S^{m}} \omega_{\iota}(q)=\operatorname{vol}\left(S^{m}\right) .
$$

Therefore, $\left\|V^{i, j}\right\|^{2}=\frac{1}{m+1} \operatorname{vol}\left(S^{m}\right)$. Now, the Proposition follows from the fact that $\left\{E^{i j} \mid 0 \leq i<j \leq n\right\}$ is an orthogonal basis of the Lie algebra of $S O(n+1)$ with respect to a multiple of the Killing form.

\section{Proof of the main result}

\subsection{The structure of $\mathcal{C}$}

H. Naitoh proved that if $M, N$ are as in Subsection 1.3 , then $(G, H)$ is a symmetric pair. We recall here the more recent and general version by $\mathrm{H}$. Tasaki. Let $\mathfrak{g}=\mathfrak{k}+\mathfrak{p}$ be the Cartan decomposition of the Lie algebra of $G$ associated to the point $o \in N$.

Theorem $4([7,11])$. Let $\mathfrak{h}$ be the Lie algebra on $H$ and let $m_{-} \subset \mathfrak{p}$ be such that $d \pi_{e} \mathfrak{m}_{-}=T_{o} M$. Then $\mathfrak{k}=\mathfrak{k}_{+}+\mathfrak{k}_{-}$and $\mathfrak{p}=\mathfrak{m}_{+}+\mathfrak{m}_{-}$in such a way that

$$
\mathfrak{h}=\mathfrak{k}_{-}+\mathfrak{m}_{-} \text {and } \quad T_{M} \mathcal{C} \cong \mathfrak{h}^{\perp}=\mathfrak{k}_{+}+\mathfrak{m}_{+} .
$$

Moreover,

$$
\left[\mathfrak{h}, \mathfrak{h}^{\perp}\right] \subset \mathfrak{h}^{\perp} \quad \text { and } \quad\left[\mathfrak{h}^{\perp}, \mathfrak{h}^{\perp}\right] \subset \mathfrak{h} .
$$




\subsection{The evolution equation for geodesics}

Let $M, N$ be as in Subsection 1.1. G. Kainz obtained in [2] a necessary and sufficient condition for a curve $f: I \rightarrow \mathcal{E}$ to be a geodesic, where $I$ is an interval of the real line.

In the very particular case when $f(t)$ is a totally geodesic embedding and $f^{\prime}(t)$ is a normal vector field along $f(t)$ for all $t \in I$, the condition simplifies as follows [6, Subsection 4.2]: $f$ is a geodesic if and only if

$$
\left.\frac{D}{d t}\right|_{t_{o}} f^{\prime}(t)(x) \in d\left(f\left(t_{o}\right)\right)_{x}\left(T_{x} M\right)
$$

for all $t_{o}$ and all $x \in M$, where $\frac{D}{d t}$ denotes covariant derivative along the curve $I \ni t \mapsto f(t)(x)$.

\subsection{The acceleration of an orbit in a normal space}

When applying the criterion above in our case, we will need an expression for the covariant acceleration of the orbit of a one-parameter group of isometries.

Let $G$ be a connected Lie group endowed with a bi-invariant Riemannian metric and let $K$ be a closed connected Lie subgroup of $G$ with Lie algebra $\mathfrak{k}$. Consider on $P=G / K$ the Riemannian metric such that the canonical projection $\pi: G \rightarrow P$ is a Riemannian submersion (the normal metric on $P)$. In these conditions, the geodesics of $G$ are one-parameter subgroups; in particular, the fibers are totally geodesic.

Lemma 5. Let $G$ and $P$ be as above, and let $\beta$ be the curve in $P$ defined by $\beta=\pi \circ \alpha$, where $\alpha(t)=\exp t(U+V)$, with $U \in \mathfrak{k}$ and $V \in \mathfrak{k}^{\perp}$. Then

$$
\frac{D \dot{\beta}}{d t}(0)=d \pi_{e}[U, V]_{e}
$$

Before proving the Lemma we recall from [9] some definitions and statements about submersions and parallel transport.

Let $\pi: B \rightarrow P$ be a Riemannian submersion with totally geodesic fibers. For $E \in T B$, let $\mathcal{H} E$ and $\mathcal{V} E$ denote the horizontal and vertical parts of $E$, respectively. The O'Neill tensor field $A$ on $B$, of type $(0,2)$, is defined by

$$
A_{E} F=\mathcal{V} \nabla_{\mathcal{H} E}(\mathcal{H} F)+\mathcal{H} \nabla_{\mathcal{H} E}(\mathcal{V} F) .
$$


Let $E$ be a vector field along a curve $\alpha$ in $B$. By the main result in [9],

$$
\mathcal{H}\left(E^{\prime}\right)=\mathcal{L}\left((d \pi(E))^{\prime}\right)+A_{\mathcal{H} E}(\mathcal{V} \dot{\alpha})+A_{\mathcal{H} \dot{\alpha}}(\mathcal{V} E)
$$

where the prime denotes covariant derivative (along $\alpha$ or $\pi \circ \alpha$, accordingly) and, if $W$ is a vector field along $\pi \circ \alpha$, then $\mathcal{L}(W)$ is the horizontal vector field along $\alpha$ projecting to $F$.

Proof of Lemma 5. We consider the Riemannian submersion $\pi: G \rightarrow P$ and apply equation (5) to $E=\dot{\alpha}=U \circ \alpha+V \circ \alpha$, whose covariant derivative vanishes since $\alpha$ is a geodesic of $G$ (the metric is bi-invariant). We obtain

$$
0=\mathcal{L}\left(\dot{\beta}^{\prime}\right)+2 A_{\mathcal{H} \dot{\alpha}}(\mathcal{V} \dot{\alpha})
$$

Hence, by definition of the tensor $A$ and using that $\nabla_{V} U=\frac{1}{2}[V, U]$ since the metric on $G$ is bi-invariant, one has

$$
\mathcal{L}\left(\dot{\beta}^{\prime}\right)=-2 \mathcal{H}\left(\nabla_{\mathcal{H} \dot{\alpha}} \mathcal{V} \dot{\alpha}\right)=-2 \mathcal{H}\left(\left(\nabla_{V} U\right) \circ \alpha\right)=\mathcal{H}(([U, V]) \circ \alpha) .
$$

Applying $d \pi$ and evaluating at $t=0$, one gets the desired formula for $\dot{\beta}^{\prime}(0)$.

Proof of Theorem 1. We consider on $\mathcal{C}=G / H$ the metric induced from $\mathcal{S}$ (which in principle may not be normal). Let $\tilde{F}: G / H \rightarrow \mathcal{E}$ be defined by $\tilde{F}(g H)=g \circ \iota$, that is, the following diagram is commutative.

$$
\begin{array}{cc}
G / H \stackrel{\widetilde{F}}{\longrightarrow} & \mathcal{E} \\
& \downarrow \Pi \\
& \mathcal{S}
\end{array}
$$

Given a geodesic $\gamma$ in $\mathcal{C}$, we will prove that $\tilde{F} \circ \gamma$ is a horizontal geodesic in $\mathcal{E}$. Hence, $F \circ \gamma$ is a geodesic in $\mathcal{S}$ and so $(\mathcal{C}, F)$ is totally geodesic, as desired. Since $F$ is $G$-equivariant and the action of $G$ preserves the metrics on $\mathcal{C}$ and $\mathcal{E}$ and also the vertical and horizontal distributions on $\mathcal{E}$ (see above their description in terms of vector fields along the embeddings), it suffices to prove the assertion only for $\gamma$ with $\gamma(0)=H$.

Now, by Theorem 4 , since the metric of $\mathcal{C}$ is $G$-invariant, the geodesics of $\mathcal{C}$ are the same as the geodesics of $\mathcal{C}$ endowed with the normal metric (see Exercise 10)b) on page 330 of [10]). Hence, $\gamma(t)=\tilde{\pi} e^{t X}$, for some $X \in \mathfrak{h}^{\perp}$. 
We call $f=\tilde{F} \circ \gamma: \mathbb{R} \rightarrow \mathcal{E}$. Now we check that we can apply the criterion of Kainz. First, $f(t)$ is totally geodesic for any $t \in \mathbb{R}$, since $f(t)=e^{t X} f(0)$, with $e^{t X}$ an isometry of $N$ and $f(0)=\iota: M \rightarrow N$, which is totally geodesic since it is reflective. Secondly, the vector field $f^{\prime}(t)$ along $f(t)$ is normal to $f(t)$. Again by $e^{t X}$-invariance, one can take $t=0$. Let $q \in M$. Since $M$ is a totally geodesic submanifold of the symmetric space $G / H$ through $o$, $q=e^{Y} . o$ for some $Y \in \mathfrak{m}_{-}$. We compute

$$
f^{\prime}(0)(q)=\left.\frac{d}{d t}\right|_{0} e^{t X} q=\left.\frac{d}{d t}\right|_{0} e^{t X} e^{Y} . o=\left.\frac{d}{d t}\right|_{0} e^{Y} e^{t Z} . o=\left(d e^{Y}\right)_{o} d \pi_{o}(Z),
$$

where

$$
Z=\operatorname{Ad}\left(e^{-Y}\right) X=\sum_{n=0}^{\infty} \frac{(-1)^{n}}{n !}\left(\operatorname{ad}_{Y}\right)^{n} X .
$$

Now, $[Y, X] \in\left[\mathfrak{m}_{-}, \mathfrak{h}^{\perp}\right] \subset \mathfrak{h}^{\perp}$ by (3). Hence, $Z \in \mathfrak{h}^{\perp}$ and so $d \pi_{e}(Z) \perp T_{o} M$. Therefore $f^{\prime}(0)(q) \perp\left(d e^{Y}\right)_{o} d \pi\left(\mathfrak{m}_{-}\right)=T_{q} M$ (this last equality is well-known to hold for totally geodesic submanifolds of a symmetric space).

Now we can use Kainz evolution equation (4). Again by $e^{Y}$-invariance, without loss of generality we may check it only at $q=o$. Let $c(t)=e^{s X} . o$ and suppose, by Theorem 4, that $X=U+V$, with $U \in \mathfrak{k}_{+}$and $V \in \mathfrak{m}_{+}$. We can apply Lemma 5 to $G / K$, with $M$ in the role of $P$ :

$$
\left.\frac{D}{d t}\right|_{0} c^{\prime}(t)=d \pi_{e}[U, V] \in d \pi_{e}\left[\mathfrak{k}_{+}, \mathfrak{m}_{+}\right]
$$

which belongs to $\mathfrak{p}($ since $[\mathfrak{k}, \mathfrak{p}] \subset \mathfrak{p})$ and also to $\mathfrak{h}$, since $\left[\mathfrak{h}^{\perp}, \mathfrak{h}^{\perp}\right] \subset \mathfrak{h}$ by $(3)$. Therefore, by (2), $\left.\frac{D}{d t}\right|_{0} c^{\prime}(t) \in d \pi_{e}\left(\mathfrak{m}_{-}\right) \in T_{o} M$, as desired.

\section{References}

[1] Bauer, M., Bruveris, M., Harms, P., and Michor, P.W., Vanishing geodesic distance for the Riemannian metric with geodesic equation the KdV-equation, Ann. Glob. Anal. Geom., 41 (2012), 461-472.

[2] Kainz, G., A metric on the manifold of immersions and its Riemannian curvature, Monatsh. Math., 98 (1984), 211-217.

[3] Kriegl, A., and Michor, P., "The convenient setting for global analysis", Surveys and Monographs 53, Providence, AMS, 1997. 
[4] Leung, D.S.P., On the classification of reflective submanifolds of Riemannian symmetric spaces, Indiana Univ. Math. J., 24 (1974), 327-339.

[5] Micheli, M., Michor, P.W., and Mumford, D., Sobolev metrics on diffeomorphism groups and the derived geometry of spaces of submanifolds, to appear in Izvestiya: Mathematics, (2012).

[6] Michor, P., and Mumford, D., Vanishing geodesic distance on spaces of submanifolds and diffeomorphisms, Doc. Math., 10 (2005), 217-245.

[7] Naitoh, H., Symmetric submanifolds and generalized gauss maps, Tsukuba J. Math., 14 (1990), 541-547.

[8] Salvai, M., Some geometric characterizations of the Hopf fibrations of the three-sphere, Monatsh. Math., 147 (2006), 173-177.

[9] O’Neill, B., Submersions and geodesics, Duke Math. J., 34 (1967), 363-373.

[10] O’Neill, B., "Semi-Riemannian geometry. With applications to relativity", Pure and Applied Mathematics 103, New York,London, Academic Press, 1983.

[11] Tasaki, H., Geometry of reflective submanifolds in Riemannian symmetric spaces, J. Math. Soc. Japan, 58 (2006), 275-297.

FaMAF-CIEM, Ciudad Universitaria, 5000 Córdoba, Argentina

salvai@famaf.unc.edu.ar 